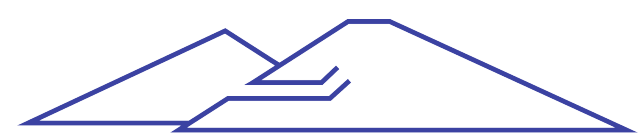

NAPOLI | IN-VENTO 2018

\title{
Mixture model in high-order statistics for peak factor estimation on low-rise building
}

\author{
F. Rigo ${ }^{1}$, T. Andrianne ${ }^{1}$ and V. Denoël ${ }^{2}$ \\ ${ }^{1}$ Wind Tunnel Lab, Dept. of Aerospace and Mechanical Engineering, University of Liège, Belgium \\ ${ }^{2}$ Stochastic and Structural Dynamics, Dept. of Urban and Environmental Engineering, University of \\ Liège, Belgium \\ Corresponding author: F.Rigo, francois.rigo@uliege.be
}

\begin{abstract}
To design reliable structures, extreme pressures and peak factors are required. In many applications of Wind Engineering, their statistical analysis has to be performed taking into account the non-Gaussianity of the wind pressures. With the increasing precision and sampling frequency of pressure sensors, large short and local peak events are more usually captured. Their relevance is naturally questioned in the context of a structural design.

Furthermore, the increasing computational power allows for accumulation and analysis of larger data sets revealing the detailed nature of wind flows around bluff bodies. In particular, in the shear layers and where local vortices form, it is commonly admitted that the Probability Density Function (PDF) of measured pressures might exhibit two or more significant components. These mixed flows can be modelled with mixture models [Cook (2016)]. Whenever several processes coexist, and when one of them is leading in the tail of the statistical distribution, as will be seen next in the context of corner vortices over a flat roof, it is natural to construct the extreme value model with this leading process and not with the mixed observed pressures. It is therefore important to separate the different processes that can be observed in the pressure histories. Once this is done, specific analytical formulations of non-Gaussian peak factors can be used to evaluate the statistics of extreme values [Kareem and Zhao (1994), Chen (2009)].

The separation of mixed processes is usually done by means of the PDF of the signals [Cook (2016)]. This information is of course essential to perform an accurate decomposition but it might be facilitated by considering higher rank information like auto-correlations and higher correlations like the triple or quadruple correlation. Indeed, the two phenomena that need to be separated and identified might be characterized by significantly different timescales, which are not reflected in the PDF. In this paper, the large negative pressures measured on a flat roof are analyzed and decomposed into two elementary processes, namely, the flapping corner vortex and the turbulent flow detaching from the sharp upstream edge.

The full paper will finally show that an accurate decomposition of the recorded pressures into their underlying modes provides a more meaningful evaluation of the extreme pressures.
\end{abstract}

\section{Introduction}

The setup made by [Blaise et al (2017)] consists of a square plan-form low-rise building (sides of $45 \mathrm{~m}$ and a height of $25 \mathrm{~m}$ ), uniformly instrumented by 121 pressure taps in a quarter of its roof (see Figure 1(a)), sampled at $500 \mathrm{~Hz}$. The model is placed at an incidence of $45^{\circ}$ in the atmospheric boundary layer test section of the wind tunnel (WT) of the University of Liege. Scaling parameters are: $\lambda_{L}=1 / 100$ (geometry), $\lambda_{U}=1 / 3.5$ (velocity) and $\lambda_{T}=1 / 28.6$ (time). Every measurement is converted in full scale. A total of $13 \mathrm{~h}$ of measurement has been taken in the WT, corresponding to $371.8 \mathrm{~h}$ full scale. This long information allows to draw precise PDF tail, necessary to study the extreme values. The flow around this low-rise building is characterized by corner vortices, that roll in a cone shape. The 2 cones on both corners have a main axis, recognizable by in the ridgelines of the standard deviation of the pressure coefficient $\sigma\left(C_{p}\right)$, in Figure 1(b). Figure 1(c) shows a map of the mean value 
of peak factor of the pressure coefficient $\mu_{g}\left(C_{p}\right)$, the highest values happening were the vortex cone touches the roof.

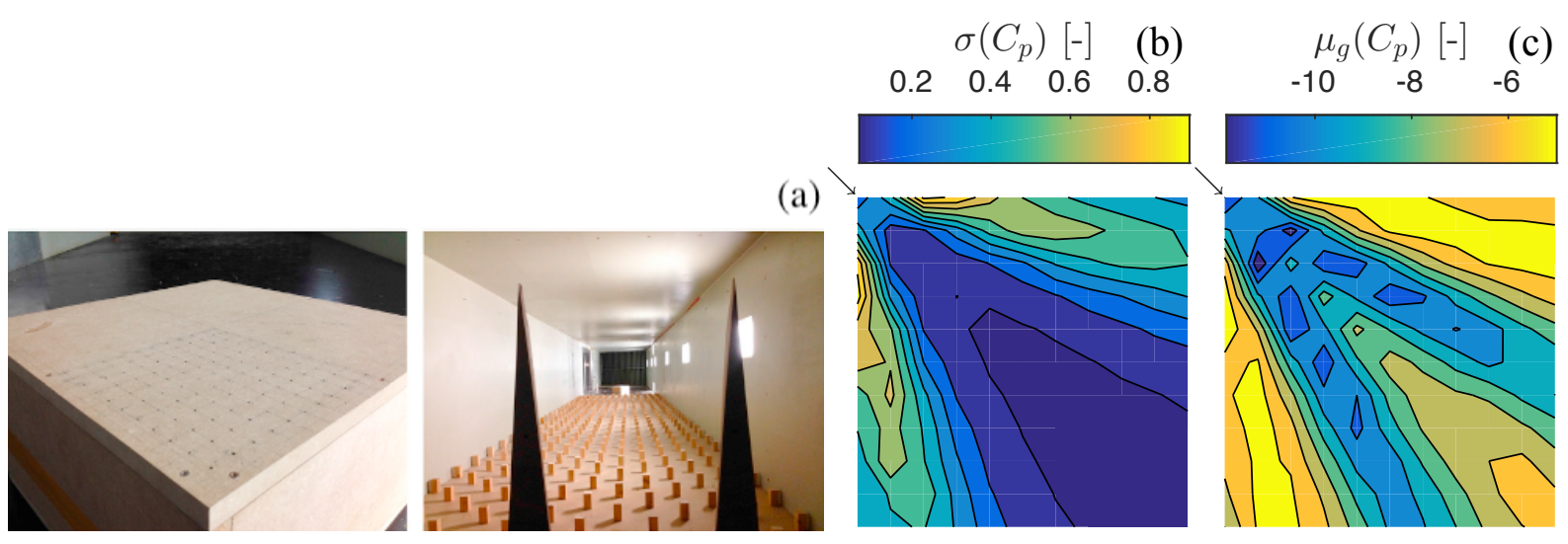

Figure 1. Model inside the WT (a), map of standard deviation (b) and peak factor (c) of pressure coefficient.

\section{Methodology}

Figure 2(a) shows the recorded pressure coefficient at tap 34 (inside corner vortices and separated region), with non-Gaussian behaviour. Two modes in the PDF are identifiable in Figures 2(b)-(c): (i) mode 1 corresponding to the turbulent background flow (as the one present on the roof region without vortex) and (ii) mode 2 to the vortices fluctuation (separated flow). Using a skew-Gaussian for the 2 modes and a skew hyperbolic secant to represent the tail, as suggested by [Cook (2016)], the PDF decomposition was simple for this tap because of its clear double bump.
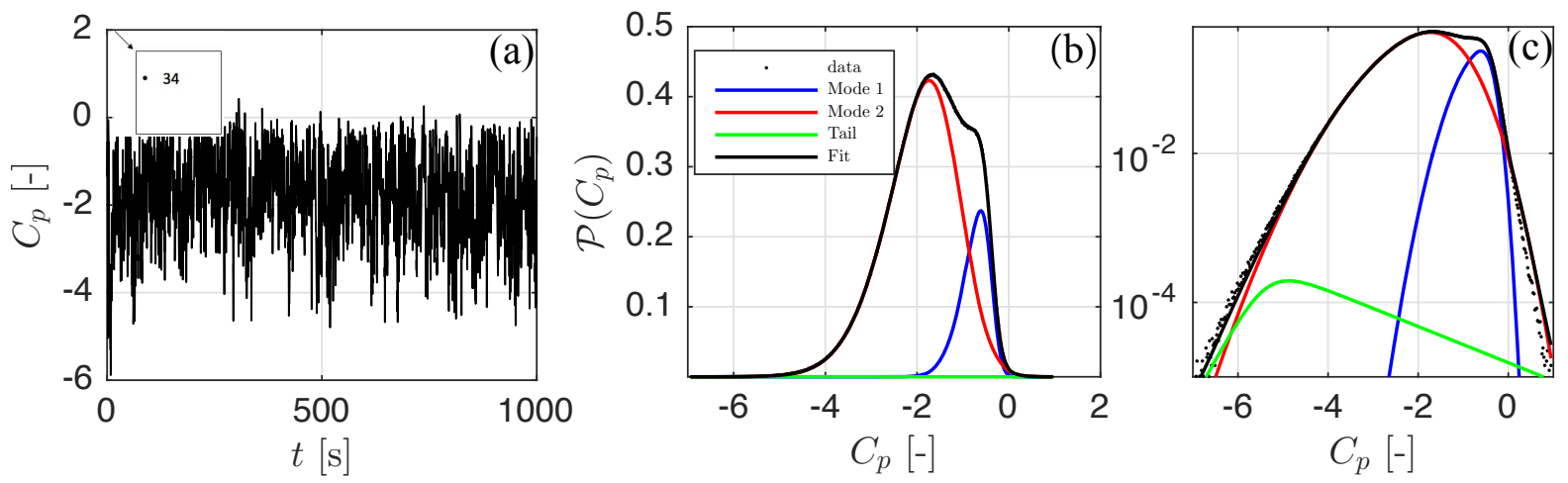

Figure 2. Time history (a), PDF in linear (b) and log (c) scale of the pressure coefficient at tap 34.

\section{Results and conclusions}

By going through the taps along the corner, the two bumps are getting closer and merge, mode 2 becoming more and more important compared to mode 1 . The method proposed by [Cook (2016)] is not robust anymore to identify initial values of the fitting algorithm. By looking at the autocorrelation function in Figure 3(b), the shape shows a break between two exponential decay functions, at around $0.4 \mathrm{~s}$. This suggests also a decomposition in 2 modes, as before: (i) the turbulent background of mode 1 with a longer memory time than (ii) mode 2 . The value of the autocorrelation at the origin is the variance. Variances of both modes are identified and are used in the PDF decomposition algorithm, to make it more robust (see Figure 3(a)). The same procedure is done using the triple correlation (with 2 delays) to decompose skewness of both modes. Figure 3(c) shows the map of the relative importance of variances of modes 1 and 2 . Mode 1 is more present at the extremities of the vortex cone. Once PDFs are decomposed, one can access the statistical properties of a given mode. It allows to correct 
the value of skewness and kurtosis used in the extreme value model of [Kareem and Zhao (1994)]. More precise estimation of peak factor is then obtained, compared to those obtained with the whole PDF, not robust in the model because of its strong non-Gaussianity.
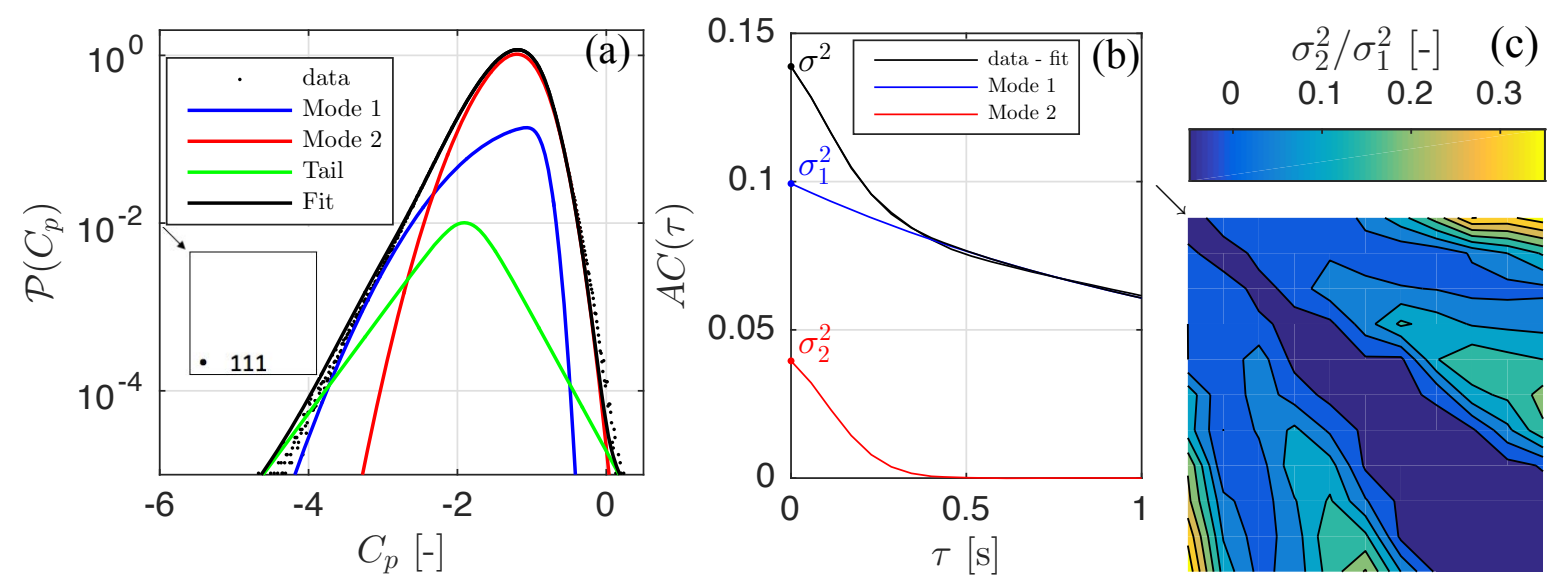

Figure 3. PDF (a) and autocorrelation function (b) of the pressure coefficient at tap 111, map of relative importance of variance of mode 2 compared to 1 (c).

\section{References}

Kareem, A., and Zhao, J. (1994). Analysis of non-Gaussian surge response of tension leg platforms under wind loads. J. offshore Mech. and Arctic Eng., ASME, 116, 137-144.

Chen, X. and Huang, G. (2009). Evaluation of peak resultant response for wind-excited tall buildings. Engineering Structures 31, 858-868.

Cook, J. (2016). Short communication: On the Gaussian-Exponential Mixture Model for pressure coefficients. Journal of Wind Engineering and Industrial Aerodynamics 153, 71-77.

Blaise, N., Andrianne, T. and Denoël, V. (2017). Assessment of extreme value overestimations with equivalent static wind loads. Journal of Wind Engineering and Industrial Aerodynamics 168, 123 133. 